Cardiovascolare, Università di Milano, Fondazione IRCCS Ca' Granda Ospedale Maggiore Policlinico di Milano, Milan, Italy.

Correspondence: S. Crotti, Terapia Intensiva "E. Vecla", Dipartimento di Anestesia, Rianimazione e Terapia del Dolore; Fondazione IRCCS Ca' Granda Ospedale Maggiore Policlinico, Via F. Sforza 35, 20122, Milano, Italy. E-mail: stefania.crotti@ policlinico.mi.it

Statement of Interest: None declared.

Acknowledgements: The authors would like to thank the entire intensive care unit staff (nurses and doctors) who fully managed veno-venous extracorporeal support and who have earned the credit for the successful and uneventful course described.

\section{REFERENCES}

1 Nava S, Hill N. Non-invasive ventilation in acute respiratory failure. Lancet 2009; 374: 250-259.

2 Plant PK, Owen JL, Elliott MW. A multicentre randomised controlled trial of the early use of non-invasive ventilation in acute exacerbation of chronic obstructive pulmonary disease on general respiratory wards. Lancet 2000; 355: 1931-1935.
3 Ward NS, Dushay KM. Clinical concise review: mechanical ventilation of patients with chronic obstructive pulmonary disease. Crit Care Med 2008; 36: 1614-1619.

4 Raghu MR, Kalpalatha KG. Review of ventilator techniques to optimize mechanical ventilation in acute exacerbation of chronic obstructive pulmonary disease. Int J COPD 2007; 2: 441-452.

5 Budweiser S, Jörres RA, Pfeifer M. Treatment of respiratory failure in COPD. Int J COPD 2008; 3: 605-618.

6 Rothpearl A, Varma AO, Goodman K. Radiographic measures of hyperinflation in clinical emphysema. Discrimination of patients from controls and relationship to physiologic and mechanical lung function. Chest 1988; 94: 907-913.

7 Gattinoni L, Pesenti A, Mascheroni D, et al. Low-frequency positive-pressure ventilation with extracorporeal $\mathrm{CO}_{2}$ removal in severe acute respiratory failure. JAMA 1986; 256: 881-886.

8 Mikkelsen ME, Woo J, Sager JS, et al. Outcomes using extracorporeal life support for adult respiratory failure due to status asthmaticus. ASAIO J 2009; 55: 47-52.

9 Cardenas VJ, Lynch JE, Ates R, et al. Venovenous carbon dioxide removal in chronic obstructive pulmonary disease. Experience in one patient. ASAIO J 2009; 55: 420-422.

10 Strueber M. Extracorporeal support as a bridge to lung transplantation. Curr Opin Crit Care 2010; 16: 69-73.

11 Olsson KM, Simon A, Strueber M, et al. Extracorporeal membrane oxygenation in nonintubated patients as bridge to lung transplantation. Am J Transplant 2010; 10: 1-6.

DOI: $10.1183 / 09031936.00021111$

\title{
Intermittent hypoxia enhances cancer progression in a mouse model of sleep apnoea
}

\section{To the Editors:}

Obstructive sleep apnoea (OSA) is a very prevalent syndrome that induces or aggravates cardiovascular, metabolic and neurocognitive disorders. Among the various challenges imposed on a patient by OSA as a consequence of apnoeas (sleep disruption, increased inspiratory efforts and recurrent hypoxaemia), intermittent hypoxia plays a major role in the pathophysiology of this sleep breathing disorder.

It has been well established that hypoxia plays an important role in regulating the various stages of tumour formation and progression [1]. Accordingly, the aim of the experimental study reported here was to test the hypothesis that high-rate intermittent hypoxia with a time course similar to that found in OSA (one or more hypoxic event per minute) enhances tumour growth. To avoid the interaction of any comorbidity, this investigation was carried out in a well-controlled animal model where the main variable under study was intermittent hypoxia.

This study, which was approved by the Ethical Committee for Animal Research of the University of Barcelona (Barcelona, Spain), was conducted in 15 pathogen-free male C57BL/6J mice (25-30 g) using a conventional murine melanoma model consisting of tumour induction by subcutaneous injection of $10^{6}$ B16F10 melanoma cells (ATCC-CRL-6475; American Type Culture Collection, Manassas, VA, USA) in the left flank region of the mouse [2]. This is a widely used cancer model in experimental research $[3,4]$, which has a response to hypoxia that is representative of a variety of cancer types $[5,6]$.

Application of chronic intermittent hypoxia (60 events $\cdot h^{-1}$ consisting of $20 \mathrm{~s}$ of $5 \% \mathrm{O}_{2}$ followed by $40 \mathrm{~s}$ of room air) started on the same day as the injection of cancer cells. Briefly, a continuous flow of gas was circulated through a box $(26 \mathrm{~cm}$ long, $18 \mathrm{~cm}$ wide and $6 \mathrm{~cm}$ high) by means of a distribution system based on multiple orifices. A pneumatic valve placed near the inlet of the box cyclically switched from the room air entrance (40 s) to a gas reservoir of hypoxic air at an oxygen fraction of $5 \%(20 \mathrm{~s})$. Seven mice were placed in the intermittent hypoxia box, with food and water available, for $6 \mathrm{~h} \cdot \mathrm{day}^{-1}$ during the light period (10:00-16:00 h) for 14 consecutive days. A normoxia control group of eight mice was placed in an identical system with the reservoir of $5 \% \mathrm{O}_{2}$ air replaced by room air. Accordingly, both groups of melanoma-injected mice were subjected to exactly the same protocol, the only difference being the breathing of normoxic or intermittently hypoxic air.

The growth of the melanoma tumour was assessed at days 8, 11 and 14 after the injection of cancer cells by externally measuring the tumour's dimensions with a calliper and estimating tumour volume as $W^{2} \times L / 2$, where $W$ is tumour width and $L$ is tumour length [2]. At day 14, the mice were sacrificed and the tumours were excised and weighed to directly measure cancer growth. 


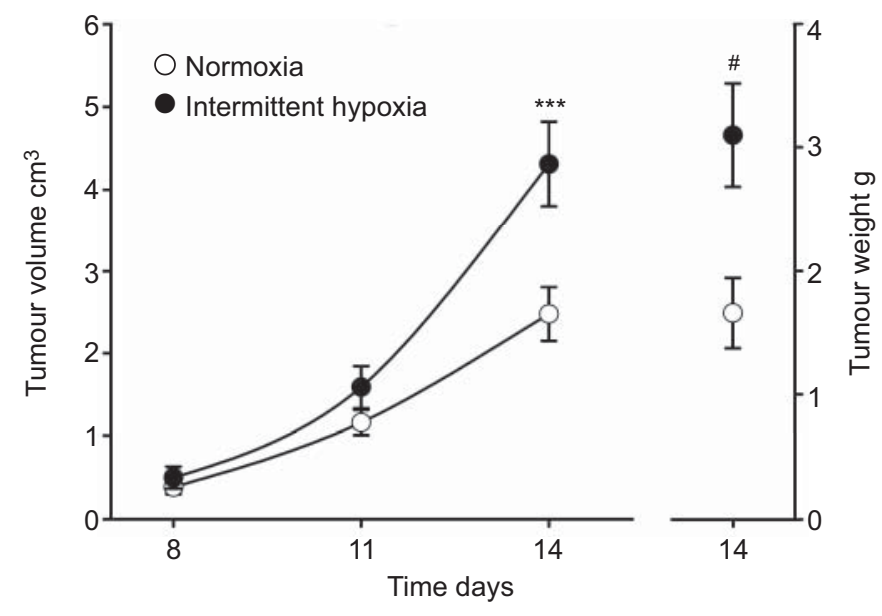

FIGURE 1. Tumour growth in both groups of mice. At day 14, tumour volume in the animals subjected to intermittent hypoxia $(n=7)$ was significantly greater ( $p<0.001$ by two-way ANOVA with Holm-Sidak post-test) than that of the normoxia group $(n=8)$. Tumour weight at day 14 was significantly greater $(p=0.012$ by unpaired t-test) in the intermittent hypoxia group than in the normoxia group. \#: $p=0.012 ; * * *: p<0.001$.

Figure 1 shows that intermittent hypoxia resulted in a two-fold increase in the growth of the melanoma tumours.

The excised tumours were fixed in formalin, embedded in paraffin blocks, cut into $4-\mu \mathrm{m}$ thick sections and stained with haematoxylin-eosin. Necrotic cells were identified by a loss of cell borders and the presence of cellular debris (fig. 2) [4]. Blind microscopic examination of a section from the central part of each animal's tumour showed a two-fold increase $(\mathrm{p}=0.080$ by unpaired $\mathrm{t}$-test) in the percentage of necrotic tumour area in the animals subjected to intermittent hypoxia (mean \pm SE $29.1 \pm 6.2 \%$ ), compared with controls $(14.5 \pm 4.7 \%$ ). Whereas in the normoxic animals, tumour necrosis tended to be more peripheral $(12.3 \pm 4.6 \%)$ than central $(2.2 \pm 0.7 \%)$ $(\mathrm{p}=0.07)$, in the animals subjected to intermittent hypoxia, this difference decreased $(17.7 \pm 7.3 \%$ and $11.3 \pm 4.9 \%$ for peripheral and central, respectively; $\mathrm{p}=0.58)$.

The potential mechanisms involved in the enhancement of cancer progression by hypoxia have been identified [1]. It is also well known that tumour growth can be modulated by the relatively slow changes (lasting from tens of minutes to hours) in the level of tissue oxygenation caused by progressive angiogenesis and irregular blood flow inside a tumour during its growth [7]. The rate of tumour growth is boosted by increased angiogenesis into the tumoural tissue and hence by augmented perfusion [8]. There is evidence that over-expression of hypoxia-inducible factor- $1 \alpha$ caused by tissue hypoxia triggers upregulation of proangiogenic mediators, such as vascular endothelial growth factor, in tumour cells [8]. Moreover, tumour progression could be enhanced by external growth factors reaching the cancer cells, in particular those systemically induced by hypoxia and hence circulating in the blood. This pathway could be of particular relevance in OSA since patients with this sleep breathing disorder exhibit increased proangiogenic factors in blood.

Although angiogenesis is augmented by hypoxia, it is known that the newly formed vascular network could present structural
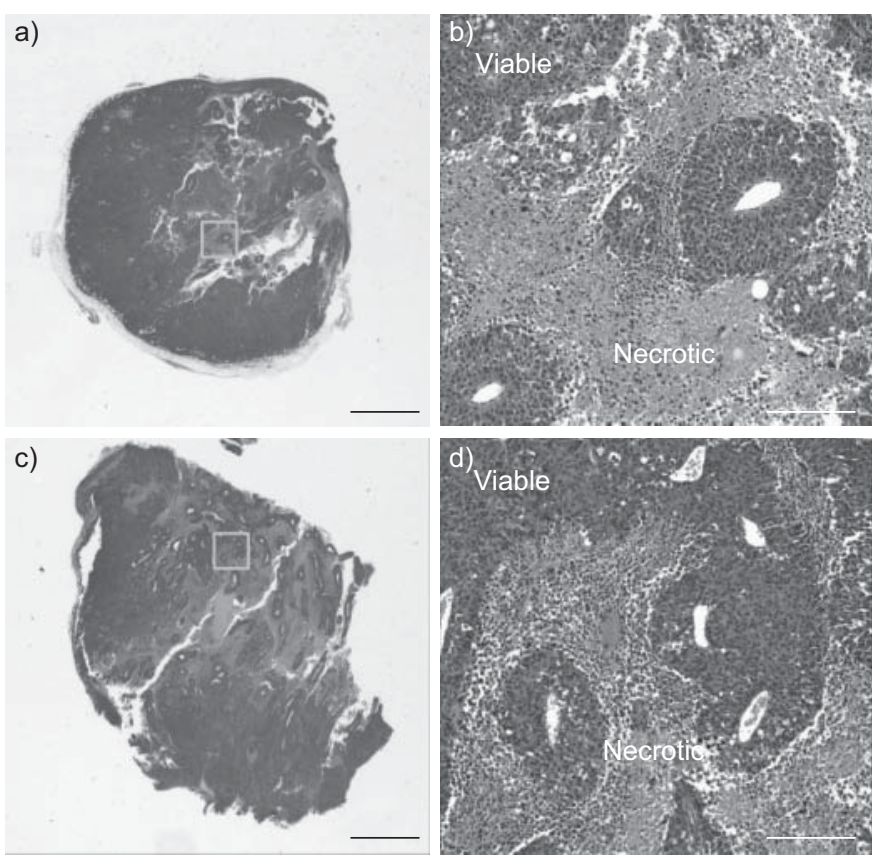

FIGURE 2. Representative tumour tissue preparations stained with haematoxylin-eosin corresponding to $a, b)$ the normoxic group and c, d) animals subjected to intermittent hypoxia. a, c) whole section of the tumour, where viable tumour areas appear darker than necrotic areas. Areas marked within a square are enlarged in b) and d), respectively. $a, c)$ Scale bars $=2 \mathrm{~mm}$; b, d) scale bars $=200 \mu \mathrm{m}$.

and functional abnormalities that lead to reduced perfusion and delivery of oxygen to the tumour tissue [9], thereby promoting tumour necrosis [10]. In fact, a higher percentage of necrotic tissue in the tumour, as we observed in the animals subjected to intermittent hypoxia, is a predictor of an aggressive cancer phenotype and a feature of tumour progression and poor prognosis [10]. However, a more detailed histological characterisation of tumour vascularisation and/or inflammation induced by intermittent hypoxia remains to be completed.

To our knowledge, the study reported here is the first one providing experimental evidence that a high-rate intermittent hypoxia mimicking the one experienced by OSA patients enhances tumour growth. Ascertaining whether there is a relationship between cancer incidence or mortality and the severity of OSA, measured by the apnoea/hypopnoea index and independent of all other relevant variables (e.g. age, obesity, smoking habits or alcohol intake), would require future studies on cohorts with a high number of patients over an extended time period. The present animal study, carried out under well-controlled conditions to investigate the effect of intermittent hypoxia, provides support for a hypothesis of a causal link between OSA and cancer.

\section{Almendros*, J.M. Montserrat ${ }^{*, \#}$, J. Ramírez , M. Torres*, J. Duran-Cantolla*,+, D. Navajas ${ }^{*, \S, f}$ and R. Farré ${ }^{*, \S}$}

*CIBER de Enfermedades Respiratorias, "Servei Pneumologia, Hospital Clínic, ${ }^{\S}$ Unitat de Biofísica i Bioenginyeria, Facultat de Medicina, Universitat de Barcelona - IDIBAPS, "Dept Anatomia Patològica, Hospital Clínic, ${ }^{f}$ Institut de Bioenginyeria de Catalunya, Barcelona, and 'Unidad de Trastornos del Sueño, 
Hospital Txagoritxu, Facultad de Medicina, Universidad del País Vasco, Vitoria, Spain.

Correspondence: R. Farré, Unitat de Biofísica i Bioenginyeria, Facultat de Medicina, Casanova 143, 08036 Barcelona, Spain. E-mail: rfarre@ub.edu

Support Statement: This work was supported in part by Ministerio de Ciencia e Innovación (grants SAF2009-02991, PI081908 and PI080277) and SEPAR.

Statement of Interest: A statement of interest for the study itself can be found at www.erj.ersjournals.com/site/misc/ statements.xhtml

Acknowledgements: The authors thank Esteve-Teijin (Barcelona, Spain) for kindly providing the gas concentrators used to prepare the gas mixtures. The authors wish to thank R. Nieto (CIBER Enfermedades Respiratorias, Bunyola, Baleares, Spain) and M.A. Rodríguez (Universitat de Barcelona, Barcelona, Spain) for their excellent technical assistance.

\section{REFERENCES}

1 Carmeliet P, Dor Y, Herbert JM, et al. Role of HIF-1 $\alpha$ in hypoxiamediated apoptosis, cell proliferation and tumour angiogenesis. Nature 1998; 394: 485-490.
2 Madi L, Bar-Yehuda S, Barer F, et al. A3 adenosine receptor activation in melanoma cells: association between receptor fate and tumor growth inhibition. J Biol Chem 2003; 278: 42121-42130.

3 Rofstad EK, Gaustad JV, Egeland TAM, et al. Tumors exposed to acute cyclic hypoxic stress show enhanced angiogenesis, perfusion and metastatic dissemination. Int J Cancer 2010; 127: 1535-1546.

4 Cormier SA, Taranova AG, Bedient C, et al. Pivotal advance: eosinophil infiltration of solid tumors is an early and persistent inflammatory host response. J Leukoc Biol 2006; 79: 1131-1139.

5 Terraneo L, Bianciardi P, Caretti A, et al. Chronic systemic hypoxia promotes LNCaP prostate cancer growth in vivo. Prostate 2010; 70: 1243-1254.

6 Yao K, Gietema JA, Shida S, et al. In vitro hypoxia-conditioned colon cancer cell lines derived from HCT116 and HT29 exhibit altered apoptosis susceptibility and a more angiogenic profile in vivo. Br J Cancer 2005; 93: 1356-1363.

7 Toffoli S, Michiels C. Intermittent hypoxia is a key regulator of cancer cell and endothelial cell interplay in tumours. FEBS J 2008; 275: 2991-3002.

8 Harris AL. Hypoxia - a key regulatory factor in tumour growth. Nat Rev Cancer 2002; 2: 38-47.

9 Vaupel P. The role of hypoxia-induced factors in tumor progression. Oncologist 2004; 9: 10-17.

10 Bachmann IM, Ladstein RG, Straume O, et al. Tumor necrosis is associated with increased $\alpha_{\mathrm{v}} \beta_{3}$ integrin expression and poor prognosis in nodular cutaneous melanomas. BMC Cancer 2008; 8: 362.

DOI: $10.1183 / 09031936.00185110$

\section{Malignant mesothelioma in Metsovo, Greece, from domestic use of asbestos: 30 years later}

\section{To the Editors:}

Malignant mesothelioma (MM) is a devastating disease, almost exclusively due to inhalation of asbestos fibres, and with higher risk for amphiboles [1]. Mediterranean regions, such as Turkey, Cyprus and Corsica, and others, such as New Caledonia [2], have experienced epidemics of MM as a result of nonoccupational, "domestic" exposure to tremolite asbestos and fibrous erionite [3]. In 1987, we reported on the very high incidence, 300 times higher than expected, of MM in the area of Metsovo, a complex of small villages in the prefecture of Ioannina in northwest Greece [4]. This, in conjunction with the previously noted very frequent pleural calcifications among Metsovites [5], led to the discovery of the culprit, a tremolite asbestos-containing whitewash ("luto" in the local dialect) [6]. The material was used by practically all households until 1940-1950. After that, it was gradually substituted by modern materials that did not contain asbestos. In 1980, luto was used by only $18 \%$ of builders and by 1990 it had been abandoned altogether (fig. 1) [7]. In 1996, we reported that the incidence of MM in the period 19851994 declined to one-third compared with the period 1980-1984, and we attributed the reduction to the abandonment of the use of luto. As a result, we concluded that the "Metsovo mesothelioma epidemic" would decline by 2020-2030 [8].
In the present update, we analyse the epidemiology of MMs after 1994 (1995-2009), compare it with that of the first 15 yrs (1980-1994), and address the question whether, 30 yrs after the first recorded case (1980), an end of the epidemic is indeed expected.

The population under study included inhabitants of the Metsovo municipality, which consists of the villages of Metsovo, Milia, Anilio and Votonossi, with a total current population of 4,417 inhabitants (2001 Greek Population Census; www.statistics.gr/ portal/page/portal/ESYE/PAGE-themes?p_param=A1620). The population of the prefecture of Ioannina, as well as the Metsovo population, has been followed-up for 30 yrs (January 1980December 2009). Taking into account the available census data within that time-frame (1981, 1991 and 2001 Greek Population Censuses), a total of 127,753 person-yrs for Metsovo, and $4,733,915$ person-yrs for Ioannina were analysed. No significant change in the population of the Metsovo and Ioannina area has occurred over those three decades since, unlike most Greek villages, there is no migration problem in Metsovo. No data were available regarding the age distribution of the particular population.

MM patients were identified through either the central registries of the two tertiary care hospitals of Ioannina or the death 